\title{
DESAFIOS DA ASSISTÊNCIA Á SAÚDE DE TRANSEXUAIS NO SISTEMA ÚNICO DE SAÚDE A PARTIR DO OLHAR DE UMA EQUIPE DE ASSISTENTES SOCIAIS
}

\author{
CHALLENGES OF TRANSEXUAL HEALTH CARE IN THE UNIQUE HEALTH \\ SYSTEM FROM DE LOOK OF A TEAM OF SOCIAL WORKERS
}

Márcia Cristina Brasil Santos ${ }^{1}$, Zélia Lima Gebrath ${ }^{2}$, Elisa Teruszkin Prestes ${ }^{3}$, Samantha Guedes Clemente Rodrigues ${ }^{4}$ e Guilherme Silva de Almeida ${ }^{5}$.

1Universidade do Estado do Rio de Janeiro, Programa de Pós-Graduação da Faculdade de Serviço Social, assistente social e Coordenadora Técnica Ambulatorial do Processo Transexualizador do Hospital Universitário Pedro Ernesto da Universidade do Estado do Rio de Janeiro, email: mcristinabrasil@hotmail.com, ORCID: https://orcid.org/0000-0002-7851-1439

2 Universidade Federal Fluminense, Programa de Pós-Graduação em Serviço Social e Desenvolvimento Regional da Faculdade de Serviço Social, Brasil, email: zeliagebrath@hotmail.com, ORCID: https://orcid.org/0000-0002-6459-1757

3 Universidade Federal Fluminense, Programa de Pós-Graduação em Serviço Social e Desenvolvimento Regional da Faculdade de Serviço Social, Brasil, email: elisaprestes.as@gmail.com, ORCID: https://orcid.org/0000-0003-0198-6986

4 Universidade Federal Fluminense, Programa de Pós-Graduação em Serviço Social e Desenvolvimento Regional da Faculdade de Serviço Social, Assistente da Sub-reitoria de Assistência estudantil da UFRJ, Brasil, email: samanthaguedes@hotmail.com, ORCID: https://orcid.org/00000002-0987-6346

5 Universidade Federal do Rio de Janeiro, Escola de Serviço Social da Universidade Federal do Rio de Janeiro, Brasil, email: gsdealmeida@gmail.com, ORCID: https://orcid.org/0000-0001-7355-6606

A R T I C LE IN F O

Article history:

Received 2020-06-01

Accepted 2020-11-30

Available online 2020-11-30
Palavras-chave: Processo transexualizador. Assistência à saúde. Contrarreforma do Estado. Desfinanciamento.

Keywords: Transsexualizing process. Health care. State counter-reform. De-financing.

RESUMO. Trata-se de reflexão sobre a assistência a saúde de pessoas transexuais no SUS, tendo como aspecto central o Processo Transexualizador - modelo assistencial que compõe a política de saúde brasileira incluindo procedimentos e condutas profissionais de diferentes graus de complexidade e diversidade técnica, como parte da assistência em saúde destinada a pessoas transexuais. Parte-se de autores vinculados ao paradigma da reforma sanitária, ao debate de gênero e ao campo marxista. $O$ estudo se deu por meio de levantamento bibliográfico e documental, bem como por observação participante. Discute-se nesse artigo os limites do programa frente à contrarreforma do Estado no âmbito da saúde, que com o sistemático desfinanciamento e desresponsabilização por parte dos governos, deixam parcelas significativas desses sujeitos à margem de cuidados básicos e com suas demandas específicas de saúde negligenciadas ou atendidas de forma insuficiente na maior parte dos estados e municípios do país. Não obstante o marco normativo que fundamenta a importância do processo transexualizador no SUS é preciso considerar que as disputas em torno da oferta pública estatal das políticas de saúde configuram uma realidade que marca a história do Brasil. Um país em que a luta pelo direito universal a saúde é uma pauta persistente e nunca plenamente contemplada. Assim, a contrarreforma do Estado nos anos 90, trará impactos para o setor saúde que irão acentuar os desafios de um campo já muito disputado, resultando em determinações limitantes para o próprio setor, e mais especificamente para as ações relativas ao processo transexualizador. 
ABSTRACT. It is a reflection on the health care of transsexual people in SUS, having as central aspect the Transexualizador Process - care model that composes the Brazilian health policy including professional procedures and conduct of different degrees of complexity and technical diversity, as part of health assistance for transgender people. It starts with authors linked to the health reform paradigm, to the gender debate and to the Marxist field. The study took place through bibliographical and documentary survey, as well as through participant observation. This article discusses the limits of the program in the face of the State's counterreform in the field of health, which with the systematic de-financing and lack of responsibility on the part of governments, leaves significant portions of these subjects to the margin of basic care and with their specific neglected health demands or insufficiently served in most states and municipalities in the country. Notwithstanding the normative framework that underlies the importance of the transsexualizing process in SUS, it is necessary to consider that the disputes over the state public offer of health policies constitute a reality that marks the history of Brazil. A country in which the fight for the universal right to health is a persistent agenda and never fully contemplated. Thus, the counter-reform of the State in the 90s, will bring impacts to the health sector that will accentuate the challenges of a field that is already very disputed, resulting in limiting determinations for the sector itself, and more specifically for the actions related to the transsexualizing process.

\section{Introdução}

Nossa intenção nesse artigo é refletir sobre os limites do processo transexualizador e da assistência à saúde de pessoas transexuais no Brasil, tentando apontar os desafios impostos ao campo da seguridade social, em especial ao Sistema Único de Saúde, em um contexto de contrarreforma do Estado num país de capitalismo dependente na atual fase imperialista do Capital. Buscamos apontar como esses determinantes (capitalismo dependente, capital imperialista e contrarreforma do Estado brasileiro) atravessam a configuração das políticas públicas de saúde, corroendo desde dentro a constituição de uma política de atenção às necessidades especificas de pessoas que se auto identificam como travestis ou transexuais, fazendo com que o processo transexualizador tenha as características que hoje apresenta.

Nessa direção é necessário considerar a formação sócio histórica e cultural brasileira tendo em vista que no Brasil as particularidades de nossa constituição enquanto sociedade é atravessada por elementos como racismo, mandonismo, patrimonialismo, elitismo, moralismo que a todo momento circulam entre os espaços públicos e privados e muitas vezes se materializam nas disputas em torno da constituição das políticas públicas, fazendo que algumas políticas "peguem" e outras não, algumas sejam privilegiadas e outras rechaçadas, como parece ser o caso das políticas com o recorte da diversidade sexual e de gênero, sobretudo, as voltadas para pessoas transexuais e travestis. 


\section{Metodologia}

O percurso metodológico para a elaboração desse artigo parte de nossa inserção enquanto assistentes sociais em equipe de saúde que atende as demandas de pessoas travestis e transexuais numa das unidades do Processo Transexualizador no Brasil. A partir dessa experiência, que se dá no Hospital Universitário Pedro Ernesto, no Rio de Janeiro, pudemos observar alguns entraves que dificultam a oferta de assistência a saúde de tais grupos, sobretudo o que tange a gestão da politica de saúde voltada para pessoas trans. Além da observação participante, nos fundamentamos em levantamento e análise bibliográficas e documentais de material que versa sobre políticas de saúde, Sistema Único de Saúde, gênero e diversidade, bem como nas experiências dos atendimentos realizados na rotina diária do trabalho.

\section{Discussão}

O desenho atual da política de atendimento a transexuais no SUS é marcado por dificuldades na materialização do marco regulatório, sendo constatável o escasso ou nenhum envolvimento de boa parte das Secretarias Estaduais e Municipais de Saúde na formulação de uma rede assistencial que dê de fato suporte às unidades habilitadas para a atenção especializada à pessoa transexual. Tais unidades de saúde acabam, por isso, isoladas e sobrecarregadas na condução dessa assistência, o que contraria a lógica da integração em rede.

A noção de rede, por sua vez, enquanto elemento fundante do SUS remete a necessidade de integração dos serviços interfederativos. Assim, uma rede de ações e serviços de saúde pressupõe conexões e integração uma vez que um serviço de saúde não conseguirá sozinho resolver as demandas que lhes chegam e que terá que contar com outros serviços de saúde (de menor ou maior complexidade), bem como com outras redes que se articulam com o setor saúde e que lhe dão suporte, como a assistência social, a educação, a de ciência e tecnologia, entre outras.

Assim, mediante da expectativa de direitos por um lado e da dificuldade de acesso, por outro, os/as usuários/as demandantes do processo transexualizador no SUS têm frequentemente acionado o sistema judiciário para terem suas requisições asseguradas. Tal 
medida, conhecida como judicialização da saúde1, gera uma série de outros problemas tanto para as unidades especializadas quanto para os/as próprios/as usuários/as, sem que haja uma responsabilização dos gestores nacional e subnacionais na condução da política nos estados e municípios. Além disso, cria-se, muitas vezes, uma animosidade dos/as usuários/as contra as unidades credenciadas e contra os/as profissionais de saúde, pois localizam nestes, a "culpa" pela não implementação plena da política e pelo não funcionamento dos serviços.

O caso do Rio de Janeiro é ilustrativo nesse sentido, na medida em que ainda não houve uma pactuação do Processo Transexualizador no Estado no sentido da construção de linhas de cuidados, fluxos de atendimento e redes de atenção envolvendo os municípios e os Gestores Estadual/municipais para que se garanta, no âmbito desse estado, a organização do atendimento às pessoas transexuais e travestis desde a atenção básica até os demais níveis de atenção.

Por parte dos municípios, respeitando-se a autonomia que dispõem para definirem suas ações de saúde na atenção primária independentemente do Gestor estadual, também não se percebe em grande parte dos municípios iniciativas de acolhimento das demandas apresentadas por esses sujeitos. Dessa maneira, não há definição das competências e dos papéis de cada ente na condução desse tipo de assistência, o que significa o não cumprimento da Política Nacional Integral de Saúde LGBT, que reitera em seu conteúdo o objetivo de "aprimoramento do processo transexualizador" (BRASIL. 2012:10).

Assim, poucos dos 92 municípios do estado do Rio de Janeiro apresentam algum tipo de atenção voltada para as necessidades especificas de saúde de pessoas transexuais e travestis. Das ações destinadas a pessoas LGBTIQ+2 como um todo, boa parte delas se concentra no campo da prevenção e cuidado em HIV/AIDS3, o que na visão dos/as próprios/as usuários/as, é estigmatizante e insuficiente.

\footnotetext{
${ }^{1}$ Para aprofundamento do tema acerca da "judicialização da Saúde" tendo como referência o SUS, ver entre outros, Mirian Ventura da Silva, que vem pesquisando e discutindo o tema tanto em sua tese de doutoramento quanto em artigos e livros recém-publicados.

2 Atualmente a sigla identitária mais utilizada para qualificar Lésbicas, Gays, Bissexuais, Travestis, Transexuais e pessoas Intersexuais é LGBTI. Em termos de movimentos sociais, uma denominação que vem ganhando força é LGBTQI +, incluindo além da orientação sexual e da diversidade de gênero a perspectiva teórica e política dos Estudos Queer. O símbolo de "mais" englobaria outras variantes identitárias. No entanto, algumas vezes nos referiremos ao movimento e a política LGBT da forma já reconhecida histórica e institucionalmente, sobretudo nos documentos oficiais, respeitando assim a história processual desse movimento

${ }^{3}$ Ao longo desse estudo, nos deteremos mais cuidadosamente sobre essa persistente vinculação entre políticas voltadas para LGBT e prevenção/combate ao HIV/AIDS. Esse não é um tema qualquer porque todas as políticas no SUS produzidas a partir dos anos 80 e 90 e voltadas à população LGBT encontram sua gênese nas políticas de enfrentamento ao HIV/Aids.
} 
Além do Rio de Janeiro, muitos outros estados da Federação enfrentam situações semelhantes sem que o Governo Central, por meio do Ministério da Saúde, crie dispositivos de indução de maior comprometimento das secretarias estaduais e municipais de saúde com o direito à saúde dessas pessoas, ainda que o movimento LGBTIQ+ tenha lutado e continue reivindicando o comprometimento do Estado frente as suas necessidades de saúde.

\section{Considerações finais}

Não obstante o marco normativo que fundamenta a importância do processo transexualizador no SUS é preciso considerar que as disputas em torno da oferta pública estatal das políticas de saúde configuram uma realidade que marca a história do Brasil. Um país em que a luta pelo direito universal a saúde é uma pauta persistente e nunca plenamente contemplada. Assim, a contrarreforma do Estado nos anos 90, trará impactos para o setor saúde que irão acentuar os desafios de um campo já muito disputado, resultando em determinações limitantes para o próprio setor, e mais especificamente para as ações relativas ao processo transexualizador, das quais reiteramos algumas:

- A ofensiva do capital no atual contexto sócio histórico brasileiro atravessa o Sistema Único de Saúde dilacerando-o e desmontando as parcas e frágeis conquistas dos movimentos sociais e trabalhadores a partir do desfinanciamento das ações voltadas para a saúde da população em geral, e em particular as ações de saúde para as populações estigmatizadas;

- O Ministério da Saúde, juntamente com os demais âmbitos das políticas setoriais são atravessados pela lógica do desmonte do bem público, pela lógica privatizante e de rentabilidade para o grande capital, restringindo dessa maneira, as ações voltadas para as necessidades reais da população.

- O Ministério da Saúde não é um espaço neutro, enfrentando suas disputas internas e sua possibilidade de influência na implementação regionalizada do Processo Transexualizador é restringida pelos limites do pacto federativo;

- Apesar da base normativa, as atribuições e competências da saúde de âmbito estadual e municipal precisam ser pactuadas nos fóruns específicos em cada Unidade Federativa (Planos Estaduais e Municipais de Saúde, Comitês Intergestores Bi-partides, entre outros) o que envolve uma intensa correlação de forças em um cenário extremamente adverso, marcado pelo ajuste econômico, pelo desfinanciamento e desresponsabilização do Estado no campo social, lembrando aqui que a pactuação das diretrizes de ação envolve investimento estatal com a política pactuada; 
- Os recursos financeiros e de pessoal das unidades de saúde são limitados para o atendimento da crescente demanda;

- O acesso de novos/as usuários/as na maioria dos programas é extremamente limitado;

- Há pouquíssimo controle social incidindo no que é desenvolvido no âmbito das unidades de saúde prestadoras de assistência à transexuais e travestis no país;

- O tempo médio de realização de modificações corporais pode ser muito superior ao que é previsto nos marcos normativo-legais (dois anos), podendo chegar há dez anos de espera por procedimentos em alguns casos.

Tais reflexões e elementos aqui dispostos configuram apenas alguns dos muitos desafios presentes na ainda escassa e frágil assistência às pessoas transexuais no sistema único de saúde. Tais problemáticas exigem dos assistentes sociais e de todos os profissionais que lutam em defesa da seguridade social e do respeito à diversidade humana um amplo e inequívoco posicionamento político em tempos de preocupantes retrocessos.

Nesse ambiente de destituição de direitos sociais e de precarização da assistência pública, a garantia do acesso a uma atenção integral em saúde, bem como as ações especificas para esses/as usuários/as se constituem um grande desafio para os amplos segmentos de sujeitos que lutam por um SUS universal e equânime, demandando uma pauta de luta que não se limite às (justas) reivindicações específicas do movimento LGBTIQ+, mas que vá além delas, alcançando a luta mais geral em busca de um sistema único de saúde para todos e radicalmente democrático.

Para tanto, urge a decifração acerca de como se configura a política de saúde num contexto societário marcado pela atual ofensiva do capital em um país de capitalismo dependente como é o caso do Brasil.

\section{Referências}

ARÁN. Márcia; Murta. Daniela; Lionço, Tatiana. Transexualidade e saúde pública no Brasil. Ciência \& Saúde Coletiva, 14(4):1141-1149, 2009.

BENTO, Berenice. O que é transexualidade. São Paulo: Brasiliense, 2008.

BOSCHETTI, I. A política de seguridade social no Brasil. In: Serviço Social: direitos sociais e competências profissionais. Brasília: 2009.

BOSCHETTI, I. et al. Política social no capitalismo - tendências contemporâneas. SP: Cortez, 2009. 
BRASIL. Conselho Nacional de Combate à Discriminação. Brasil Sem Homofobia: Programa de combate à violência e à discriminação contra GLTB e promoção da cidadania homossexual. Brasília: Ministério da Saúde, 2004.

BRASIL. Lei Orgânica da Saúde n.8.080 de 19 de setembro de 1990. Disponível em: http://portal.saude.gov.br/portal/arquivos/pdf/lei8080.pdf. Acesso em agosto de 2018.

BRASIL. Ministério da Saúde PORTARIA № 807, DE 21 DE MARÇO DE 2017.

BRASIL. Ministério da Saúde. Portaria n.1.707 de 18 de agosto de 2008. Diário Oficial da União. Poder Executivo, Brasília, DF,19 ago. 2008. Seção 1, p.43. Institui no âmbito do SUS o processo transexualizador a ser implantado nas unidades federadas, respeitadas as competências das três esferas de gestão.

BRASIL. Ministério da Saúde. Portaria n.2.836 de 01 de dezembro de 2011. Diário Oficial da União. Poder Executivo, Brasília, DF, 02 dez. 2011. Seção 1 n.231, p.37.Institui, no âmbito do Sistema Único de Saúde (SUS), a Política Nacional de Saúde Integral de Lésbicas, Gays, Bissexuais, Travestis e Transexuais (Política Nacional de Saúde Integral LGBT).

BRASIL. Ministério da Saúde. Portaria n.457 de 19 de agosto de 2008. Diário Oficial da União. Poder Executivo, Brasília, DF, 20 ago. 2008. Seção 1, p. 69. Aprova a regulamentação do processo transexualizador no âmbito do SUS.

BRASIL. PORTARIA № 2.803, DE 19 DE NOVEMBRO DE 2013.

BRASIL. PORTARIA № 2.836, DE 1 DE DEZEMBRO DE 2011. Política Nacional de Saúde Integral LGBT).

BRAVO, M. I. S. e MENEZES, J. S. B. A saúde nos governos Lula e Dilma: algumas reflexões. In: Saúde na atualidade: por um sistema único de saúde estatal, universal, gratuito e de qualidade. 1. ed. - Rio de Janeiro: UERJ, Rede Sirius, 2011. 76 p.

Maria I S. SERVIÇO SOCIAL E REFORMA SANITÁRIA: lutas sociais e práticas profissionais. SP: Cortez, 1996;

IRINEU, B. MELLO, L. FROEMming, C. Políticas Públicas de trabalho, Assistência Social e Previdência social para a População LGBT no Brasil: sobre desejos e realizações. Revista de Ciências Sociais, Fortaleza, v. 44, n. 1, jan/jun, 2013, p. 132-160

B. 10 Anos do Programa Brasil Sem Homofobia: notas críticas. REVISTA TEMPORALIS. Brasília (DF), ano 14, n. 28, p. 193-220, jul./dez. 2014

KUSCHNIR. Rosana; Lima. Luciana Dias de; Faria. Baptista. Tatiana Wargas de; Machado. Cristiani Vieira. Configuração da rede regionalizada e hierarquizada de atenção à saúde no âmbito do SUS. In Qualificação de gestores do SUS. / Organizado por Roberta Gondim de Oliveira, Victor Grabois e Walter Vieira Mendes Júnior. - Rio de Janeiro, RJ : EAD/ENSP, 2009.

MELLO L. et al. Por onde andam as Políticas Públicas para a População LGBT no Brasil. ReVISTA SOCIEDADE E ESTADO, V. 27, N. 2, MAIO 2012. 
POLIGNANO, M. C. História das políticas de saúde no Brasil: uma pequena revisão. CADERNOS DO INTERNATO RURAL - Faculdade de Medicina/UFMG, 2001. Disponível em: file:///C:/Users/pc33/Downloads/historia-das-politicas-de-saude-no-brasil-[16-030112SES-MT].pdf. Acesso em 15 de setembro de 2018.

SANTOS. S.M. de M. dos. Direitos, Desigualdades e Diversidades. In: Política social no capitalismo - tendências contemporâneas. SP: Cortez, 2009.

SOARES, A. SANTOS. NR. Financiamento do Sistema Único de Saúde nos governos FHC, Lula e Dilma. SAÚDE DEBATE: RIO DE JANEIRO, V. 38, N.100 P. 18-25, JAN-MAR 2014

SOARES, Raquel Cavalcante. A contrarreforma na política de saúde e o SUS hoje: impactos e demandas ao serviço social. Tese (Doutorado) Universidade Federal de Pernambuco. CCSA. Serviço Social, 2011.

TEIXEIRA. F.B. Dispositivos de dor? Poderes que (con)formam as transexualidade. 1. Ed. São Paulo: Annablume/FAPESP, 2013.

TEIXEIRA, Sônia Fleury. Saúde e democracia: a luta do CEBES (org.) São Paulo: Lemos Editorial, 1997.

VIEIRA, F. S., SÁ E BENEVIDES, R. P. de. O Direito à Saúde no Brasil em Tempos de Crise Econômica, Ajuste Fiscal e Reforma Implícita do Estado. Revista de Estudos e Pesquisas sobre as Américas V.10 N.3, 2016 ISSN: $1984-16391$. file://C:/Users/usuario/Desktop/EC\%2095\%20e\%20os\%20impactos\%20na\%20saude\%20pu blica.pdf 